\title{
O conhecimento dos trabalhadores sobre a importância do uso do equipamento de proteção individual para a saúde auditiva
}

\author{
The workers' knowledge about the use of the equipment of individual protection for the \\ health hearing \\ Rachel Costa Façanha1 ${ }^{1}$ Geice Ribeiro Azevedo²
}

\begin{abstract}
RESUMO: A Perda Auditiva Induzida pelo Ruído (PAIR) é do tipo neurossensorial sem características de reversão e por muitas vezes por apresentar-se bilateral. Esse tipo de perda não costuma ultrapassar $40 \mathrm{~dB}$ em frequências baixas e $75 \mathrm{~dB}$ em altas. $\mathrm{O}$ presente estudo teve como objetivo descrever o conhecimento dos funcionários sobre a importância do uso do equipamento de proteção auditiva individual, os benefícios que trazem a sua utilização e os malefícios decorrentes do não uso constante. Este estudo foi de natureza descritiva, transversal com enfoque quantitativo, com coleta de dados efetuada no período entre julho e agosto de 2014. Foi aplicado um questionário antes e após uma palestra sobre conservação auditiva, contendo variáveis relacionadas ao conhecimento dos trabalhadores sobre a importância do uso de equipamento de proteção auditiva individual, que teve como público-alvo 70 funcionários da área operacional de uma empresa no ramo de bebidas, DIBRASA LTDA, em Pinheiro Maranhão. Os resultados apontaram que 57,1\% não fazem uso do equipamento de proteção auditiva e apenas $21,4 \%$ já realizaram teste auditivo. $78,5 \%$ não têm conhecimento sobre o ruído e o que ele pode causar. 45,7\% tiveram alguma orientação sobre a utilização do equipamento de proteção auditiva e $67,1 \%$ acham que o equipamento poderá trazer benefícios ao longo de sua rotina de trabalho. Concluiu-se que a grande maioria não faz uso do equipamento de proteção individual e não tem conhecimento sobre o que o ruído poderá causar, havendo uma necessidade de implantação e implementação de um programa de conservação auditiva por parte da empresa.
\end{abstract}

PALAVRAS-CHAVE: Saúde Auditiva; Equipamento de Proteção Auditiva Individual; Perda Auditiva Induzida pelo Ruído (PAIR).

ABSTRACT: The Induced Hearing Loss (NIHL) is sensorineural without reversal characteristics and often by presenting itself up bilateral. This type of loss usually does not exceed $40 \mathrm{~dB}$ at low frequencies and $75 \mathrm{~dB}$ at high. The present study aimed to describe the employees' knowledge about the importance of using individual hearing protection equipment, the benefits it brings to their use and the damage arising from the inconsistent use. This study was descriptive, cross with quantitative approach, with data collection in the period between July and August 2014. A questionnaire was applied before and after a lecture on hearing conservation, containing variables related to employees' awareness of the importance of using individual hearing protection equipment, that targeted a public of 70 employees of the operational area of a company in the beverage industry, DIBRASA LTDA in Pinheiro, Maranhão. The results showed that $57.1 \%$ do not use hearing protection equipment and only $21.4 \%$ performed hearing test. $78.5 \%$ are unaware about the noise and what it can cause. $45.7 \%$ had some guidance on the use of hearing protection equipment, and $67.1 \%$ think that the equipment can provide benefits throughout their work routine. It was concluded that the vast majority does not make use of personal protective equipment and is not aware of what the noise can cause. So there is a need for establishment and implementation of a hearing conservation program by the company.

KEYWORDS: Hearing Health; Individual Hearing Protection Equipment; Induced Hearing Loss (NIHL).

${ }^{1}$ Mestra em Saúde Coletiva pela Universidade Federal do Maranhão. Professora no Curso de Fonoaudiologia e

Coordenadora do Curso Especialização em Audiologia - UNICEUMA. E-mail: rachel.facanha@ceuma.br

${ }^{2}$ Graduação em Fonoaudiologia pela Universidade CEUMA. E-mail: melo_geise @ hotmail.com 


\section{INTRODUÇÃO}

Anatomicamente o ouvido é dividido em ouvido externo, médio e interno, onde o ouvido externo capta o som, levando-o ao ouvido médio que se propaga na membrana timpânica responsável pela transmissão das ondas sonoras, finalizando no ouvido interno onde está localizada a cóclea que contém líquido e células ciliadas que se movem através das vibrações sonoras e é responsável pelo equilíbrio (DE PAULA, 1999).

O conceito de ruído é descrito de acordo com o que se segue na prática da Higiene do Trabalho, sendo um fenômeno físico vibratório sem característica definida que se propaga no ar em uma dada frequência e com diferentes pressões (MESSIAS, 2001). Alterações metabolicas e mecânicas podem facilmente ser determinadas pela exposição contínua ao ruído, onde o maior prejuízo se dá nas células ciliadas (NUDELMANN et al, 2001).

Sabe-se que o ruído em grande intensidade e por um longo período de tempo poderá ocasionar uma perda auditiva e que ele está em terceiro lugar dos maiores agentes causadores de doenças ocupacionais Trabalhador exposto ao ruído tem mais incidência em adquirir uma perda, por não usar devidamente o equipamento de proteção auditiva (EPA) ou pela ineficácia do mesmo (MESSIAS, 2001).

Os protetores usados pelo funcionário devem reduzir o nível de ruído ao qual estará exposto. Existem os protetores de inserção que devem ser adequados ao canal auditivo e os do tipo concha que são de fácil colocação (NUDELMANN et al, 2001).

Ferreira Júnior (1999) fala que a utilização adequada do equipamento de proteção individual (EPI) deverá seguir fatores necessários como a facilidade em manuseá-lo, a colocação e manutenção do equipamento, o conforto do funcionário, custo e a qualidade do produto (GRECA E MARTINS, 2010).

A Fonoaudiologia exerce grande influência no que se refere à saúde auditiva do trabalhador, buscando a conscientização do uso do equipamento de proteção e aplicando medidas preventivas para evitar a exposição dos trabalhadores ao ruído. Deve haver uma preocupação maior quando há a inserção de máquinas no ambiente de trabalho e é nesse momento em que entra o profissional capacitado, para que possa estar cuidando da acústica e da saúde dos trabalhadores (CONSELHO REGIONAL DE FONOAUDIOLOGIA, 2010).

Os índices de perda auditiva induzida pelo ruído (PAIR) vêm-se multiplicando e se tornando uma das maiores doenças relacionadas ao trabalho. Além do ruído outros fatores podem contribuir para uma possível perda, como mudanças no clima, agentes químicos, intolerância a sons intensos e 
vibrações. Também poderá apresentar os chamados sintomas extra-auditivos que são dor de cabeça, alterações no sono, tontura, problemas digestivos, irritação, entre outros. É importante para o trabalhador tomar conhecimento sobre esses tipos de riscos para que o mesmo possa estar ciente dos cuidados que deverá tomar. Deve-se entender que a PAIR não acontece da noite para o dia, ela chega de forma progressiva em forma de zumbidos e quando há dificuldade em compreender determinados sons como a fala (NUDELMANN et al, 2001).

A implementação do Programa de Conservação Auditiva - PCA tem o objetivo de minimizar o surgimento de risco quanto a PAIR. Os programas devem ser inseridos nas empresas a fim de manterem as informações corretas aos funcionários e aos seus empregadores, visando o conforto do funcionário quanto ao uso do seu equipamento e a estabilidade da empresa. O EPA deve estar de acordo com o ambiente ao qual se destina para que seu uso seja aceito pelo funcionário, com o modelo adequado para a atenuação do ruído. Alguns não fazem o vedamento adequado, o que possivelmente acarretará alterações auditivas (CONSELHO REGIONAL DE FONOAUDIOLOGIA, 2010).

A Norma Regulamentadora (NR15), da Portaria MTB n³ 3.214/1978 (BRASIL, 1978), delimita que o máximo do limite de exposição ao ruído contínuo ou intermitente seja de $80 \mathrm{~dB}$ por 8 horas diárias para funcionários que não utilizam protetores auriculares, e a exposição a níveis de ruído a $115 \mathrm{~dB}$ (ruído de impacto) só poderá ser feita quando o funcionário estiver devidamente protegido (FEDERAL, 2014).

Não há tratamento ou cura para a PAIR, pois a mesma ainda é considerada irreversível. Por isso o melhor meio é a prevenção. A instalação do uso do equipamento de proteção individual (EPI) deverá ser feito de forma rígida pela empresa e compromisso do funcionário. Isso seria um grande passo para evitar o grande número de trabalhadores acometidos por perdas e afastados do mercado de trabalho (MESSIAS, 2001).

A realização de ações educativas como palestras envolvendo os riscos sobre a falta de proteção, os sintomas ocasionados e os benefícios do uso do EPA podem ser benéficos a fim de esclarecer dúvidas quanto ao assunto abordado. Portanto, a intervenção fonoaudiológica dentro da empresa traz eficácia para a conservação da saúde auditiva do trabalhador (SILVA, 2008).

A presente pesquisa teve como objetivo descrever o grau de conhecimento dos trabalhadores quanto ao uso do equipamento de proteção auditiva individual e os benefícios e malfícios quanto a sua não utilização. 


\section{MATERIAL E MÉTODOS}

Foi realizado um estudo de caráter descritivo, observacional e quantitativo, realizado na empresa DIBRASA LTDA, na cidade de Pinheiro/MA.

Foram incluídos na pesquisa todos os funcionários do setor operacional e que trabalham há mais de dois anos na empresa com carga horária de 8 horas por dia, independentemente de sexo ou idade, que são indicados a usar o Equipamento de Proteção Auditiva e todos aqueles que assinaram o Termo de Consentimento Livre e Esclarecido que atende à Resolução no 466/2012 do Conselho Nacional de Saúde, autorizando a participação no presente estudo.

A não inclusão nesta pesquisa está voltada aos funcionários do setor administrativo e aqueles que já apresentam perda auditiva.

Foram entregues aos funcionários dois questionários contendo o mesmo conteúdo, com 20 questões objetivas, abordando sobre a utilização do equipamento de proteção auditiva, questões sobre a exposição contínua ao ruído e o conhecimento dos funcionários sobre o tipo de equipamento usado na empresa tendo o mesmo conteúdo nos mesmos, elaborado pela pesquisadora.

Iniciaram-se com o preenchimento do primeiro questionário pelos funcionários, para avaliarlhes o grau de conhecimento antes da palestra educativa. Após, foi dado início à palestra que teve como temas a conservação auditiva, benefícios e malefícios da utilização do equipamento de proteção individual.

A palestra contou com a participação do Técnico de Segurança do Trabalho da empresa, que a iniciou fazendo as apresentações. Ao término da palestra foi solicitado aos funcionários que respondessem o segundo questionário de acordo com o que teriam aprendido ou ficado esclarecido durante a palestra.

Os dados coletados e analisados surgiram a partir das respostas obtidas nos questionários 1 e 2 com respostas objetivas em sim e não; desta forma, esses resultados foram passados o software Epi Info para determinar a quantidade exata de respostas positivas para o uso do Equipamento de Proteção Auditiva e o grau de conhecimento sobre a perda auditiva.

No final da palestra não houve perguntas, apenas algumas considerações dadas pelo técnico de segurança do trabalho da empresa.

O trabalho foi encaminhado para o Comitê de Ética da Plataforma Brasil, com aprovação em maio de 2014. 


\section{RESULTADOS E DISCUSSÃO}

A pesquisa foi realizada com 70 funcionários todos do sexo masculino que trabalham no setor operacional, os quais necessitam usar o EPA. Observam-se na tabela 1 as respostas ao primeiro questionário:

Tabela - 1 Distribuição das respostas antes da realização da palestra

\begin{tabular}{|c|c|c|}
\hline Item & $N F$ & Sim \\
\hline Faz uso do equipamento de proteção auditiva? & 30 & $42,8 \%$ \\
\hline Realizou teste auditivo? & 15 & $21,4 \%$ \\
\hline Tem conhecimento sobre o ruído e o que ele pode causar? & 15 & $21,4 \%$ \\
\hline Teve alguma orientação sobre a utilização do equipamento de proteção auditiva? & 32 & $45,7 \%$ \\
\hline Acha que o equipamento pode trazer benefícios ao longo de sua rotina de trabalho? & 47 & $67,1 \%$ \\
\hline Pretende usar o equipamento de forma correta e nos horários em que estiver exposto ao ruído? & 33 & $47,1 \%$ \\
\hline Participou de palestra sobre saúde auditiva e a mesma esclareceu suas duvidas? & 54 & $77,1 \%$ \\
\hline
\end{tabular}

*NF - número de funcionários

Dos 70 funcionários, apenas 30, totalizando 42,8\%, fazem uso do equipamento de proteção auditiva. Apenas 15 (21,4\%) já realizaram teste auditivo e têm conhecimento sobre o ruído e o que ele pode causar. $45,7 \%$ tiveram alguma orientação sobre a utilização do equipamento de proteção auditiva, 67,1\% acharam que o equipamento poderá trazer benefícios ao longo de sua rotina de trabalho, $47,1 \%$ fazem uso do equipamento de forma correta nos horários de exposição ao ruído e $77,1 \%$ participaram de palestra sobre saúde auditiva e a mesma esclareceu suas dúvidas.

Com base na tabela 1, observamos que o número de funcionários conscientes sobre a utilização do uso do equipamento de proteção auditiva ainda não totaliza a grande maioria e que apenas 15 deles já fizeram testes auditivos o que deveria ser primordial quanto à admissão na empresa.

A pesquisa feita por Gleisi em 2007 mostrou que, dos 100 funcionários analisados, todos concordaram que a exposição ao ruído pode prejudicar a sua audição, o que discorda desta pesquisa, pois dos 70 apenas 15 tem consciência do risco que estão sujeitos. A mesma pesquisa foi feita sobre o uso do equipamento de proteção, 86 (86\%) fazem uso do equipamento de proteção e somente 30 participantes (42,8\%) assinalaram sim para esta questão (VIVIAN, 2007).

Em pesquisa já realizada, no que se refere à participação do funcionário em palestras sobre saúde auditiva, os resultados corroboram os achados desta pesquisa. 54 funcionários $(77,1 \%)$ não participaram de palestras sobre saúde auditiva, ou seja, o que deveria ser uma atividade constante na empresa, não é de fato realizado (VIEIRA, 2000). 
De acordo com a pesquisa realizada pelo Centro de Especialização em Fonoaudiologia Clinica - CEFAC, dos 40 funcionários, 25 fazem uso contínuo, o que me leva a crer que as duas pesquisas aproximam-se dos resultados, mostrando que pouco mais da metade concorda com o seu uso (CONSELHO REGIONAL DE FONOAUDIOLOGIA, 2010).

Tabela 2 - Distribuição das respostas após palestra educativa

\begin{tabular}{l|cc}
\hline \multicolumn{1}{c|}{ Item } & NF & Sim \\
\hline $\begin{array}{l}\text { Tem conhecimento sobre o ruído e o que ele pode causar? } \\
\text { Teve alguma orientação sobre a utilização do equipamento de proteção } \\
\text { auditiva? }\end{array}$ & 70 & $100 \%$ \\
$\begin{array}{l}\text { Acha que o equipamento pode trazer benefícios ao longo de sua rotina de } \\
\text { trabalho? }\end{array}$ & 62 & $100 \%$ \\
$\begin{array}{l}\text { Pretende usar o equipamento de forma correta e nos horários em que estiver } \\
\text { exposto ao ruído? }\end{array}$ & 65 & $92,8 \%$ \\
$\begin{array}{l}\text { Participou de palestra sobre saúde auditiva e a mesma esclareceu suas } \\
\text { duvidas? }\end{array}$ & 70 & $100 \%$ \\
\hline
\end{tabular}

NF* Número de Funcionários

Dos dados levantados neste segundo questionário, vemos que, após a palestra educativa, todos se dizem conscientes sobre os efeitos do ruído e sobre a orientação quanto ao uso do equipamento de proteção auditiva, ou seja, $100 \%$.

Para os achados relacionados aos benefícios que o equipamento pode trazer a sua rotina de trabalho, vemos que $62(88,5 \%)$ concordaram positivamente a esta questão. $65(92,8 \%)$ pretendem fazer uso do equipamento de proteção nos horários de trabalho e 70 (100\%) afirmaram participar de palestra de conservação auditiva e que a mesma esclareceu suas dúvidas.

De acordo com um estudo realizado pela Universidade Tuiuti do Paraná, os resultados obtidos sobre a pretensão do funcionário quanto ao uso do equipamento de proteção, $75 \%$ concordaram plenamente e $21 \%$ disseram apenas concordar, com isso traz semelhanças aos resultados obtidos nesta pesquisa, onde foram obtidos $65(92,8 \%)$ concordando em fazer uso do mesmo (VIVIAN, 2007).

Com base na mesma pesquisa, $96 \%$ estão convencidos de que o protetor auditivo pode proteger a sua audição e evitar a perda auditiva, assemelhando-se ao que foi referido nesta quanto a estarem conscientes sobre o efeito do ruído e sobre a orientação ao uso do equipamento de proteção auditiva, onde obtivemos $100 \%$ para as questões após a palestra educativa (VIVIAN, 2007).

Segundo Svensson, em pesquisa realizada na Suécia em 2004, foi identificado que, dos 100 funcionários analisados, $85 \%$ acreditavam que o protetor auditivo poderá evitar perdas auditivas, o que se assemelha a esta pesquisa, quando foi citado que $62 \%$ acreditavam que o equipamento poderá trazer benefícios a sua rotina de trabalho (MORATA, 2008). 


\section{CONCLUSÃO}

Observou-se que houve uma mudança significativa com relação ao conhecimento e importância do uso do equipamento de proteção individual, sendo demonstrado no questionário aplicado, após palestra educativa, um nível maior de respostas positivas para sua utilização.

Grande parte desses funcionários não apresentou e não demonstrou interesse com relação aos possíveis problemas ocorrentes a não utilização do EPI, mas conclui-se que, após as orientações dadas pela pesquisadora, eles tiveram mais interesse em se adaptar ao uso contínuo do equipamento no ambiente de trabalho, o que consequentemente os previnirá em relação à perda auditiva. Com base nesses achados, vemos a necessidade de implantação e implementação de um programa de conservação auditiva por parte da empresa.

\section{REFERÊNCIAS}

DE PAULA, U.S. Ruído Riscos e Prevenção. 3rd ed. São Paulo: Hucitec; 1999.

MESSIAS, T.S. Manual Prático de Avaliação e Controle do Ruído. 2nd ed. São Paulo: LTR; 2001.

NUDELMANN, A.A; DA COSTA, A.E; SELIGMAN, J; IBAÑEZ, R.N. PAIR- Perda

Auditiva Induzida Pelo Ruído. 2nd ed. Rio de Janeiro: Revinter; 2001.

GRECA, A.L; MARTINS, M.. Conhecimento dos Trabalhadores de uma empresa de

Manutenção Industrial em relação à Proteção Auditiva. São paulo: 2010.

CONSELHO REGIONAL DE FONOAUDIOLOGIA.Centro de Referência em Saúde do

Trabalhador do Estado do Rio de Janeiro. [Online].; 2010 [cited 2014 Abril 23. Available from:

HYPERLINK "http://www.crefono1.gov.br. Disponível em:

http://crefono1.gov.br/img/Arq/Downloads/CFFa_n\%C2\%BA_162_-_2014_-

_Envia_Boletins_da_Fiocruz_sobre_PDVRT/BOLETIM\%20n\%C2\%BA\%201,\%20JAN-

ABRIL\%202010.pdf .

FEDERAL, G. Ministério do trabalho e Emprego. [Online].; 1997/2008 [cited 2014 Abril 23.

Available from: HYPERLINK 1. Disponivel em: http://portal.mte.gov.br/legislacao/normaregulamentadora-n-15-1.htm.

SILVA, C.B. Avaliação do Conhecimento de Trabalhadores sobre medidas de Controle após Treinamento de Conservação Auditiva. 2008. 
VIVIAN, A.G. Conhecimento dos Trabalhadores de uma Empresa de Alimentos sobre

Ruído e Perda Auditiva. UTP-Universidade Tuiuti do Paraná.Curitiba, 2007.

VIEIRA, G.R. Equipamento de Proteção Auditiva: UM estudo na Empresa de

Florianópolis. In. Itajaí:CEFAC: Centro de Especialização em Fonoaudiologia Clínica; 2000

MORATA, T.C. Conhecimento de Trabalhadores sobre Ruído e seus Efeitos em Indústria Alimentícia. International Archives of Otorrinolaryngology. vol.12. n.01. Janeiro/Março 2008. 